\title{
Design and Application of the RFID Technology in ERP
}

\author{
Juan Tian, Xiujuan Wang \& Lanhua Zhang \\ College of Information Engineering, Taishan Medical University, Tai'an 271016, China
}

\begin{abstract}
The application of the RFID (Radio Frequency Identification) technology establishes the base for the realization of the quick automation of ERP (Enterprise Resources Planning). Starting from the application background, the application of the RFID technology is discussed in this article, and the structure and characteristics of the RFID technology applied in ERP are analyzed. Combining with the concrete example, the system and module of the use of RFID are designed, and the application of the module and the realization mode of the interface are introduced in detail.
\end{abstract}

Keywords: ERP, RFID, Label

\section{Introduction}

WTO brings new opportunities and higher requirements for the development of various industries of China, especially for the China manufacturing with cheap production and resources. Both hopes and gaps have been found by Chinese enterprises, the domestic and international competition make all enterprises realize that they must strengthen their strengths, including the hardware and the software which also influences and restrains the development of enterprise. The continual enhancement of the information technology, the gradual perfect of the networking degree, and the management and the application of the enterprise informationization become the focus concerned by all enterprises again. For the soul of the manufacturing automation, the ERP has the selection with low risk and high return which is needed by enterprises, and when times enters into the automation stage from the mechanization stage, the use of the automation equipments has largely enhanced the disposal quantity and speed of the operation information, and the RFID technology is one of necessary functional module.

Starting from the application actuality of RFID, the applications in the ERP are designed and implemented in this article, combining with relative technologies of ERP, and the concrete example is used to validate these applications.

\section{Actuality and analysis}

\subsection{Application background}

Because of the low cost advantages of the production factors such as human resource, China has been the global manufacturing center, and transnational companies move their manufacturing plants to China one after another, but the new research indicates that the supply chain cost including carry and logistics is very high, which may even exceed the low cost advantage of the manufacturing, so enterprises must manage by the information system, realize the client relation management with higher service level, possess the ability of modernization management with large scale, high added value, and quick speed, and the new information management system could realize exact information and real-time inquiry, and complete the construction of the supply chain information system, where the quick service is one of important characteristics of the system.

By virtue of different concrete environments, each enterprise needs to establish its own ERP, but the characteristics and requirements of the system make the software go to the currency development in the design. Based on the barcode technology, the RFID application system has been proved as one important module to complete above functions and fulfill above requirements in the practice.

The mutual infiltration and improvement of the RFID technology and the barcode technology have changed the structure and nature of traditional products and by the identification and compiling function of the video recognizer, the combination with the ERP system could be realized successfully.

\subsection{Feasibility analysis}

Because the RFID technology has many advantages such as advancement, adaptability, simple operation, and quick efficiency, its application range is very extensive, such as the auto automatic identification and checking, the electric ticket, the special qualification certificate management, the commodity anti-counterfeiting, the EPC supply chain management, the Barrier free passage, the small commodity checking, and the special equipment management checking. In most countries, the development key of the RFID technology has been pushed to the production automation, the financial trade internationalization, and the production management supply chain. For ERP, the warehousing, the deliver, and the checking of the products in the manufacturing should be managed 
by the computer. Taking the warehousing as the example, the label will be affixed to each cargo space in the warehouse, marking the number of the goods, and when these goods enter into the warehouse, the portable video recognizer will be used to scan the number and quantity of the goods, and then it will be connected with the host computer, and input the collected data to the host computer which will dispose these data. By endowing a unique label to each goods in the circulation and acquiring the information of this label in the circulation, the information of the product will be more exact and complete. To enhance the management quality, the system will contain the whole process from leaving the factory to the selling in the market, and the RF communication makes the ERP real-time data disposal be possible, and the new system could optimize the flow, and omit and change most traditional flows, so the application of the RFID technology in the ERP is the feasible and necessary step.

\subsection{System structures and characteristics}

The RFID technology is the automation technology combining the auto-measurement technique with the identification technology, using some carrier to disposal the information.

As a kind of high-efficiency technology in the auto identification, the measurement technology exerts active function in the life, and aiming at different industries and characteristics, various measurement technologies emerge as the time requires. The application of various measurement technologies enhances the automation degree of the system. In the measurement technology, the general flow is very simple, i.e. adopting the measurement equipment to acquire signals first, then disposing these signals, and finally applying these signals to realize the high-efficiency activities by certain implementation institution. In the same way, the development of the logistics technology has been largely pushed when applying the measurement technology in various parts of the logistics. For example, in the loading process, the use of the measurement technology checking the goods will reduce the waiting time before loading, and enhance the veracity of the data and save human resource. In the storage process, the measurement technology could exert the substitute function, and for the checking and registering of goods before warehousing, and the arrangement of the goods position in warehousing, and the record of the goods in delivering, the auto-measurement technology could largely save the human and material resources, and enhance the logistics efficiency. For the quick pick-up of goods in delivery, the checking and registering of goods in delivery, and the inquiry of the storage information, especially the checking of the storage information, the auto-measurement technology could not be replaceable. The application of the auto identification technology makes the measurement technology become possible, so the auto identification technology mainly disposes various physical signals. In the practice, corresponding identifications will be made aiming at various usual signals, with the function of read and write, which is mainly realized by the scanning of the wireless view to the physical signals and the corresponding conversion. In the material following, carry tools, and goods identification, the RFID technology has been applied extensively, and it is a kind of practical and highly-efficient technology.

Except for the development and use of the RFID application module, the barcode technology and the RF technology, the ERP system generally includes software, computer hardware, RF equipment, and other peripheral equipments, and the important one is the data joint between the RFID application module and other basic modules.

\section{Design and implementation}

\subsection{System design}

Based on the barcode technology, the RFID application system is composed by the label, the identification equipment, the computer system, and the communication system. According to different concrete practice environments and application systems, the collocations of RFID are different.

The whole system is mainly used to realize the real-time acquisition of data, complete the commodity standard management according to the automation degree of commodities, and complete standard managements such as cargo place, palletizing, and warehouse management according to the practice.

In the automation management of cargos, the real-time collection, storage, and disposal of the commodity information should be completed by the wireless mode, and taking the RFID technology as the core, the wireless network communication and information system will be fully utilized to complete the real-time acquisition and storage of the data.

The standardization of the warehouse management requires that the warehousing, deliver, and various operations and spot disposals of cargos must fulfill the automation requirements, and the label affixing, the truck specification, and the cargo sequence should be allocated reasonably. 


\subsection{Environment explanation}

The implementation of the system by the RFID technology is mainly divided into two parts, and the one is the implementation of the function module running after the RF portable terminal logs, and the other is the server software with the functions such as storage management and statistics. In the usual storage management system of ERP, the front-end could use the development language closely combining with the database to realize the functions such as storage management and statistics, and for the RF terminal, on the one hand, the software along with the factory could be used to manage the data and complete the inception of the data in the system, and on the other hand, the system could recompile the terminal until the acquisition of the terminal data is directly completed. The selection of the structure should be disposed according to the demand of enterprise, mainly referring the real-time requirements of the enterprise data and the requirement of the enterprise information resource.

\subsection{Design of RFID module}

For the enterprises with different use ranges and automation degrees, they should select different modules to complete the combination of the automation equipment with the ERP software. For usual ERP software, two modules can be designed to realize the joint between RFID with the system, and the one gives priority to the system, and it compiles the terminal equipment of RFID according to the data format and the operation characters described in the system, and the other one is to automatically receive and store the fixed data in the terminal equipment according to the function model provided by the terminal equipment, and then analyze and disposal the data format and characteristics for the application of the system.

\subsection{Application of function module}

After the data joint mode is set, the combination with ERP is the correct design of the RF technology to realize the real-time automation. Taking the storage management information as the example, the function demands of RFID in the system could be seen in Figure 1.

In this application, the RFID is mainly applied in four parts, i.e. the data acquisition when warehousing, checking, and delivering, and various data inquiries.

In the inquiry operation, the warehousing data is provided by the cargo label information collected by RF, and then the terminal equipment will store the data according to the interface disposal function, and the collector is connected with the computer, and the collected information will be inputted into the computer and saved in the database to complete the warehousing, and the warehouse management program will further disposal the data. The delivery data are compared and analyzed with the data in the warehouse according to the terminal data. The checking of data is to mainly analyze the delivery information according to the warehousing information, and other operations will be implemented according to the warehousing information and similar warehousing operation flows.

\subsection{Interface design}

According to the module design of the system, the interface program should be design to realize the real-time information disposal of the RFID and the information system. In the module design, two modes could compile the interface, and the portable terminal could complete the functions such as data storage, disposal, and communication transmission, and when communicating with the PC, the terminal could communicate with the computer by the interface program and the series link. The management and disposal of data mainly include the input and output of data. The inputs of data will be different according to different module design modes, and the interface design giving priority to the system could directly read the data from the terminal, and link the data with the database directly, and realize the data operation. The interface design giving priority to the equipment could save the data as the files or independent data formats according to the formats provided by the equipment, and complete the operation of the files to realize the storage and search of the database.

\section{Conclusions}

In the ERP system, the RFID technology could be used to dispose the data about cargo conveniently, and effectively complete the combination with the system. In the supply chain system, the RFID technology is more and more important. The wireless real-time data acquisition and disposal could largely enhance the efficiency of the operation of the storage logistics center in the manufacturing and the information system, and significantly enhance the use efficiency of data by other modules in the system, and provide powerful guarantee for the management concept of ERP.

As the advanced concept of the ERP enterprise management, the application of RFID only starts. And in the 
practice, because of the standard and economic factors, the comprehensive application and enhancement need to be further improved and implemented. The RFID application module in the system could realize the basic demand of enterprise to the ERP system, and the function modules with higher automation degree and stronger adaptability should be updated continually.

\section{References}

Craig. S. Mullins, interpreted by Li, Tianzhu, Ren, Jianli \& Xiao, Yanqin. (2003). Practice and Process: Database Management. Beijing: Electronic Industry Press.

Dong, Zhonglang \& Ou, Lin. (2000). The Development of the Modern Depot Management and the Application of the RF Technology. MM Electromechanical Information. No.10.

Hatch, Melanie L. Badinelli, Ralph D. (1999). Concurrent optimization in designing for logistics support. European Journal of Operational Research. No. 115(1). May. P.77-97.

Huang, Tiyun. (2003). Management Information System. Beijing: Higher Education Press.

Wei, Qiang, Wu, Yaohua \& Zhang, Lanhua. (2005). Design and Application of Barcode Technology in WMS. Computer Engineering and Design. No.11.

Wu, Jian \& Shi, Zhong-Ke. (2004). Optimization model of the area logistics system. Journal of South China University of Technology (Natural Science). No. 32(8)32. August. p 68-71.

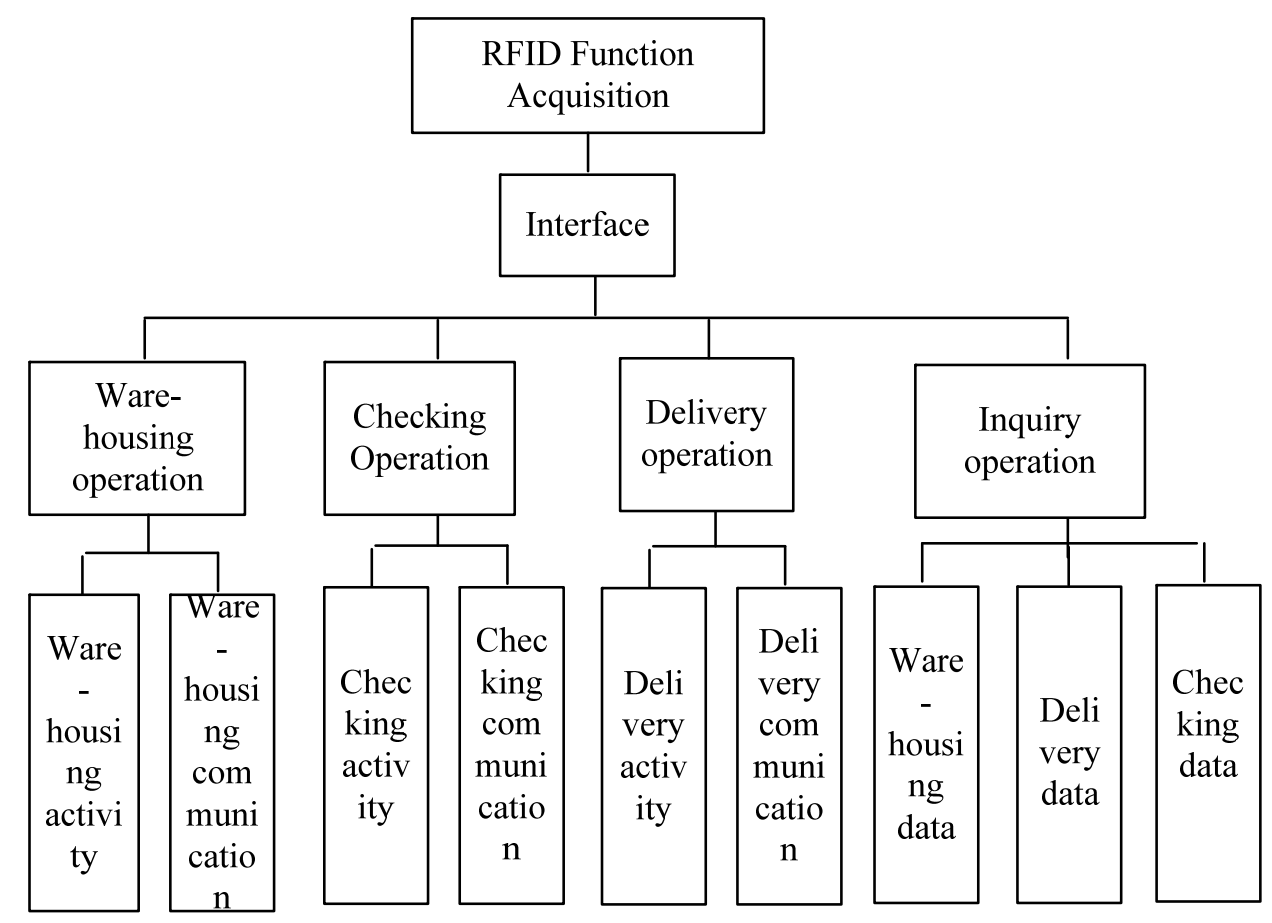

Figure 1. Applications of the Data Acquisition Function 